\title{
Methyl group dynamics in glassy toluene: A neutron scattering study
}

\author{
A. J. Moreno, ${ }^{\text {a) }}$ A. Alegría, and J. Colmenero \\ Departamento de Física de Materiales y Centro Mixto CSIC-UPV/EHU. Universidad del País Vasco (UPV/ \\ EHU), Apartado 1072, 20080 San Sebastián, Spain \\ M. Prager and H. Grimm \\ Institut für Festkörperforschung des Forschungszentrums Jülich, D-52425 Jülich, Germany \\ B. Frick \\ Institute Laue-Langevin, BP 156X, F-38042 Grenoble, France
}

(Received 30 May 2001; accepted 5 September 2001)

\begin{abstract}
We present a neutron scattering study on methyl group dynamics in glassy toluene. The spectra in the whole temperature range, covering the transition from quantum rotational tunneling to classical hopping, have been successfully analyzed in terms of a potential barrier distribution model. The average barrier in the glass is found to be notably higher than the unique barrier of the crystalline $\beta$-phase, whose short-range structure is known to be similar to that of the glass. Due to the mainly intermolecular origin of the interactions on the methyl groups in toluene, it is concluded that the rotational potentials are strongly affected by structural disorder. (C) 2001 American Institute of Physics. [DOI: 10.1063/1.1413742]
\end{abstract}

\section{INTRODUCTION}

The rotational dynamics of methyl groups is generally driven by an effective one-dimensional single-particle potential. ${ }^{1-3}$ The intensity of the potential can be determined to first order from the strong dependence of the rotational tunneling frequency, $\hbar \omega_{t}$, on the potential barrier height. ${ }^{1-3}$ In order to determine the potential shape, the measurement of the tunneling frequency is usually complemented by measurements of the librational levels in the phonon spectrum and the classical hopping rate at high temperature. ${ }^{4}$ Due to the mainly intermolecular origin of the hindering potential in many systems, methyl groups are often used as internal probes to obtain information about the different contributions to the local field ${ }^{5-7}$ and characteristics of the environment as disorder. ${ }^{8-10}$

Incoherent neutron scattering is an especially adequate technique for this kind of study, since the tunneling frequency of a great variety of compounds lies in the $\mu \mathrm{eV}$ range, ${ }^{3}$ being directly observable as two sharp, resolution limited, inelastic lines at $\pm \hbar \omega_{t}$ (neutron energy gain and loss). ${ }^{1-3}$ Moreover, selective deuteration allows one to increase the contrast for the rotor hydrogens with respect to other atoms. This property is especially useful for studies in organic compounds (the incoherent cross section for proton is 80 barns $=80 \times 10^{-24} \mathrm{~cm}^{2}$, while it is only 2 barns for deuterium, 0.5 barns for nitrogen, and 0 for carbon and oxygen).

Local disorder yields distributions of barriers for methyl group rotation. ${ }^{8-10}$ As a consequence, each individual methyl group contributes with a different spectrum, so that the resulting spectrum is given by a superposition of single-methyl (or "crystallike") spectra. This effect is especially pronounced in strongly disordered systems as structural glasses, where such distributions are very broad, and due the limited

${ }^{a}$ Electronic mail: wabmosea@sq.ehu.es energy resolution of the neutron scattering spectrometers, lead to spectra without defined tunneling peaks. ${ }^{11,12}$ The absence of such peaks makes it difficult to determine the values of the rotational barriers in the glass. The recently introduced rotation-rate-distribution-model (RRDM) ${ }^{10-16}$ which involves only three parameters (see below), allows such a determination. A series of neutron scattering studies on polymers has confirmed the suitability of this model. ${ }^{10-15}$

Methyl group dynamics in low molecular weight glasses is especially interesting. In contrast to polymers, it is possible to make a direct comparison with the crystalline state, and some insight may be obtained on the effect of structural disorder on the intermolecular contribution to the rotational barrier. A first study has been performed recently on sodium acetate trihydrate. ${ }^{16}$ The average barrier in the glass has been found to be, within the experimental error, equal to that of the crystal $(403 \mathrm{~K}){ }^{17}$

In comparison with sodium acetate trihydrate, which barrier presents an important intramolecular contribution, ${ }^{18}$ toluene is a more interesting system to study the effects of disorder on methyl group dynamics, since its barrier is basically of intermolecular origin. ${ }^{19}$ Moreover, it can be easily obtained in the glassy state (glass transition temperature $T_{g}$ $\approx 117 \mathrm{~K})^{20}$ with moderate cooling rates and it can be partially deuterated in order to attenuate the incoherent scattering from the ring hydrogens. With these ideas in mind, we have carried out a thorough investigation of methyl group dynamics in glassy ring-deuterated toluene by means of incoherent neutron scattering, covering the whole temperature range from quantum rotational tunneling to classical hopping. Complementary neutron diffraction measurements were carried out in order to determine the corrections to the total scattering function due to coherent scattering. Moreover, the coherent static intensity was also determined for a fully protonated sample of glassy toluene in order to obtain 
information about the carbon-carbon correlations and to be compared with the structural information of the crystalline phase.

The paper is organized as follows: In Sec. II we briefly summarize the usual model for methyl group dynamics in crystalline systems and we outline the basic ideas of the RRDM. In Sec. III we give experimental details. In Secs. IV and $\mathrm{V}$ we present and discuss the neutron scattering measurements in glassy toluene in terms of the RRDM. Finally, conclusions are given in Sec. VI.

\section{THEORETICAL ASPECTS}

\section{A. Crystalline systems}

The usual single-particle model for methyl group rotation in crystalline systems at liquid helium temperatures is that of a rigid rotor tunneling through a one-dimensional rotational potential $V(\Phi)$. This potential is required to have the rotational symmetry of the methyl group. Usually, only the threefold term of the Fourier expansion is retained. The corresponding Hamiltonian is ${ }^{1-3}$

$$
H=-B \partial^{2} / \partial \Phi^{2}+V_{3}(1-\cos 3 \Phi) / 2
$$

with $B$ the rotational constant. For a protonated methyl group, $B=0.655 \mathrm{meV}^{3}$ The level scheme of $H$ consists of a series of librational levels in the meV range which are tunnel-split by the overlapping of the neighboring wave functions into sub-levels with symmetry $A$ and $E$, the latter consisting of a degenerate doublet $\left(E_{a}, E_{b}\right)$. These labels correspond to the irreducible representations of the symmetry group $C_{3}$. The frequency $\omega_{t}=E_{t} / \hbar$, with $E_{t}$ the energy splitting of the ground librational level, is commonly referred to as the "tunneling frequency." Neutron scattering can access tunneling frequencies from $\hbar \omega_{t} \approx 0.5 \mu \mathrm{eV} \quad\left(V_{3}\right.$ $\approx 700 \mathrm{~K}$ ) to the free rotor line at $\hbar \omega_{t}=B$. The spectrum consists of two inelastic peaks centered at $\pm \hbar \omega_{t}$, resulting from transitions $A \leftrightarrow E$ and an elastic peak resulting from both, transitions $E_{a} \leftrightarrow E_{b}$, and transitions with no change of symmetry. The incoherent scattering function for rotational tunneling in a threefold potential, normalized to scattering from one hydrogen, is ${ }^{1-3}$

$$
\begin{aligned}
S_{\mathrm{MG}}^{\mathrm{inc}}(Q, \omega)= & \frac{5+4 j_{0}(Q r)}{9} \delta(\omega)+\frac{2\left[1-j_{0}(Q r)\right]}{9} \\
& \times\left[\delta\left(\omega+\omega_{t}\right)+\delta\left(\omega-\omega_{t}\right)\right],
\end{aligned}
$$

with $\hbar Q$ the momentum transfer and $\hbar \omega$ the energy transfer of the neutron. $j_{0}$ is the zeroth-order spherical Bessel function, and $r$ the $\mathrm{H}-\mathrm{H}$ distance in the methyl group ( $r$ $=1.78 \AA){ }^{10}$

When increasing temperature, a transition or "crossover" from the regime of quantum tunneling to classical hopping is driven by the coupling of the methyl group to the lattice phonons. $^{21,22}$ The tunneling peaks broaden into Lorentzian lines and shift towards the central elastic line. ${ }^{1-4}$ These inelastic Lorentzians involve $A \leftrightarrow E$ transitions. ${ }^{21,22}$ At the same time, the same part of the elastic scattering involving transitions $E_{a} \leftrightarrow E_{b},{ }^{21,22}$ transforms into a Lorentzian quasielastic line around the elastic peak. ${ }^{1-4}$ The temperature dependence of these inelastic and quasielastic broadenings is given by complicated expressions involving coupling constants of the rotor to the lattice modes, and sums of Bose factors over the phonon frequencies resonant in the librational energies. ${ }^{21,22}$ Such expressions can be well approximated by an Arrhenius law ${ }^{1-4}$ driven by the first librational energy $E_{01}$, i.e.,

$$
\Gamma_{i(q)}=\gamma_{i(q)} \exp \left(-E_{\mathrm{br}} / k T\right),
$$

with $E_{\mathrm{br}} \approx E_{01}$. Indices $i, q$ refer, respectively, to inelastic and quasielastic lines. The shift of the tunneling peaks is determined in a similar way, though in this case the sum is done over the whole phonon spectrum, ${ }^{21,22}$ resulting in a lower activation energy, $E_{S}$, though close to $E_{01}$

$$
\hbar \Delta \omega_{t}=\gamma_{s h} \exp \left(-E_{s} / k T\right) .
$$

In these equations, $\Gamma_{i}, \Gamma_{q}$ are the half-width at halfmaximum (HWHM) of the inelastic and quasielastic Lorentzians respectively, and $\hbar \Delta \omega_{t}$ the shift of the maximum of the inelastic Lorentzians. $\gamma_{i}, \gamma_{q}$, and $\gamma_{s h}$ are temperatureindependent preexponential factors. The incoherent scattering function for the crossover regime is ${ }^{23}$

$$
\begin{aligned}
S_{\mathrm{MG}}^{\mathrm{inc}}(Q, \omega)= & \frac{1+2 j_{0}(Q r)}{3} \delta(\omega)+\frac{2\left[1-j_{0}(Q r)\right]}{9} \\
& \times\left[L\left(\omega ; \Gamma_{q}\right)+L\left(\omega+\left(\omega_{t}-\Delta \omega_{t}\right) ; \Gamma_{i}\right)\right. \\
& \left.+L\left(\omega-\left(\omega_{t}-\Delta \omega_{t}\right) ; \Gamma_{i}\right)\right] .
\end{aligned}
$$

The end of the transition to the classical hopping regime is characterized by the merging of the inelastic and quasielastic Lorentzians into a single quasielastic Lorentzian, which broadens according to an Arrhenius law ${ }^{1-4}$

$$
\Gamma=\Gamma_{\infty} \exp \left(-E_{A} / k T\right),
$$

with $E_{A}$ the activation energy for classical hopping, defined as the difference between the top of the barrier and the ground state. $\Gamma_{\infty}$ is a temperature-independent preexponential factor. The merging takes place in a narrow temperature interval $(\Delta T \approx 7-10 \mathrm{~K})$ typically around $\approx 50 \mathrm{~K}$. In the following, we will neglect the width of this interval, and we will represent it as a unique temperature, $T_{c}$, that will be referred to as the "crossover temperature," that marks the onset of the classical behavior. The incoherent scattering function for the classical hopping regime is ${ }^{1}$

$$
\begin{aligned}
S_{\mathrm{MG}}^{\mathrm{inc}}(Q, \omega)= & \frac{1+2 j_{0}(Q r)}{3} \delta(\omega) \\
& +\frac{2\left[1-j_{0}(Q r)\right]}{3} L(\omega ; \Gamma) .
\end{aligned}
$$

It is straightforward to see that the crossover function (5) reduces to Eqs. (2) and (7) in the two temperature limits. At liquid helium temperatures $\Gamma_{i}, \Gamma_{q}, \Delta \omega_{t} \rightarrow 0$, recovering Eq. (2). At high temperatures, where the classical limit has been reached, the tunneling peaks are shifted to zero $\left(\Delta \omega_{t}=\omega_{t}\right)$, recovering Eq. (7) if $\Gamma_{i}$ and $\Gamma_{q}$ are now substituted by the classical $\Gamma$. 


\section{B. Rotation-rate-distribution-model}

In a glass, the different local environments yield a different barrier $V_{3}$ for each methyl group, and therefore, a barrier distribution $g\left(V_{3}\right)$. As a consequence, the spectrum of the glass results from a superposition of the crystallike spectra of the individual methyl groups, weighted by $g\left(V_{3}\right)$. Thus, the corresponding incoherent scattering function for methyl group dynamics in a glass is obtained as

$$
S_{\mathrm{RRDM}}^{\mathrm{inc}}(Q, \omega)=\int g\left(V_{3}\right) S_{\mathrm{MG}}^{\mathrm{inc}}\left(Q, \omega, V_{3}\right) d V_{3},
$$

where the individual functions $S_{\mathrm{MG}}^{\mathrm{inc}}\left(Q, \omega, V_{3}\right)$ are defined according to Eqs. (2), (5), and (7).

In a glass, due the superposition of the individual spectra, the shift and broadening of the individual peaks and their merging when reaching the classical regime is not observable. For this reason, it is not evident which physical description (the crossover picture below $T_{c}$, or the classical one above $T_{c}$ ) must be taken for each methyl group at the respective temperature. This ambiguity can be removed by introducing a functional relationship between the crossover temperature for each methyl group and its barrier height $T_{c}$ $=T_{c}\left(V_{3}\right)$. The latter is obtained by taking into account that the classical behavior for a given methyl group will be reached when its rate for incoherent hopping becomes comparable to its rate for coherent tunneling. Thus, we define $T_{c}$ as the temperature where $\Gamma / \hbar \omega_{t}=1$ or, from Eq. (6)

$$
k T_{c}=E_{A} / \ln \left(\Gamma_{\infty} / \hbar \omega_{t}\right) .
$$

As $E_{A}$ and $\hbar \omega_{t}$ are obtained as functions of $V_{3}$ from the eigenvalues of $H, T_{c}$ depends only on the barrier height $V_{3}$ and on the preexponential factor $\Gamma_{\infty}$. This latter parameter is taken as barrier-independent in good approximation (see Sec. V).

As shown elsewhere, ${ }^{14}$ we make the approximation $\gamma_{i}$ $=\gamma_{q}$, and denote both factors as $\gamma_{\mathrm{br}}$. We further make the approximation $E_{S}=E_{01} \cdot{ }^{14}$ Experiments show typical ratios of $\Gamma_{q} / \Gamma_{i} \approx 0.5$ and $E_{S} / E_{01} \approx 0.7$. However, the simplifications introduced here seem reasonable in glassy systems, since such fine effects are hidden in the broad distributions of these parameters. We impose a ratio of 1 simply for "aesthetics" reasons.

Now we see that the parameters $\gamma_{i}, \gamma_{q}, \gamma_{s h}$ for each methyl group can also be expressed as functions of only $V_{3}$ and $\Gamma_{\infty}$. Thus, as required by the continuity condition $\Gamma_{i(q)}\left(T_{c}\right)$ $=\Gamma\left(T_{c}\right)$, we have

$$
\gamma_{\mathrm{br}}=\Gamma_{\infty} \exp \left[\left(E_{01}-E_{A}\right) / k T_{c}\right] .
$$

As in the classical onset the tunneling frequency will be shifted to zero, we have

$$
\gamma_{s h}=\hbar \omega_{t} \exp \left(E_{01} / k T_{c}\right) .
$$

By following the shown procedure, we only need to know $\Gamma_{\infty}$ and the parameters of the distribution $g\left(V_{3}\right)$ to evaluate unambiguously all the quantities appearing in Eqs. (2)-(7), and therefore, the integral of Eq. (8) in the whole temperature range. Finally we add the incoherent contribution of the other atoms of the molecule and the coherent contribution, both assumed to be elastic

$$
\begin{aligned}
S(Q, \omega)= & \sigma_{\mathrm{coh}} S(Q) \delta(\omega)+\left(\sigma_{\mathrm{inc}}-\sigma_{\mathrm{inc}}^{\mathrm{MG}}\right) \delta(\omega) \\
& +\sigma_{\mathrm{inc}}^{\mathrm{MG}} S_{\mathrm{RRDM}}^{\mathrm{inc}}(Q, \omega),
\end{aligned}
$$

with $\sigma_{\text {coh }}$ and $\sigma_{\text {inc }}$ the total coherent and incoherent cross sections and $\sigma_{\text {inc }}^{\mathrm{MG}}$ the incoherent crossection of the three hydrogens of the methyl group. $\sigma_{\mathrm{coh}} S(Q)$ is the coherent static intensity. The function (12) is convoluted with the instrumental resolution for comparison with the experimental scattering function $S_{e}(Q, \omega)$.

\section{EXPERIMENT}

Samples of ring-deuterated toluene purchased from Chemotrade, Cortec, and MDS were used in the experiment. The ratio of deuteration was beyond $98 \%$ in all of them. The scattering cross sections per ring-deuterated toluene molecule are $\sigma_{\text {coh }}=72.1, \sigma_{\text {inc }}=251.1$, and $\sigma_{\text {inc }}^{\mathrm{MG}}=240.8$ barns.

Neutron scattering measurements in the quasielastic range were carried out at the backscattering spectrometers IN16 at the Institute Laue-Langevin (ILL, Grenoble, France) and BSS at the Institut für Festkörperforschung des Forschungszentrums (IFF, FZ Jülich, Germany), and at the timeof-flight spectrometer IN5 at the ILL. Diffraction measurements were taken at D7 (ILL). A hollow cylindrical sample of a radius of $1.80 \mathrm{~cm}$ and a thickness of $0.2 \mathrm{~mm}$ was used in the IN16 measurements. Flat samples of thicknesses of 1.0 and $0.6 \mathrm{~mm}$ were used at BSS, and at IN5 and D7, respectively. The corresponding transmissions were close to $90 \%$, allowing to neglect multiple scattering effects in the $Q$-range we restricted our analysis to, (1.3-1.9) $\AA^{-1}$. The glassy state of toluene was achieved by quenching the sample into liquid nitrogen. The instrumental resolutions were calibrated by vanadium samples, which show purely elastic scattering. The raw data were corrected for detector efficiencies, scattering from the sample holder and absorption by means of the standard programs of the ILL and IFF.

The scattering angle $\Phi$, incident neutron beam energy $E_{0}$, momentum transfer $Q$ and energy transfer $\hbar \omega$ of the scattered neutrons are related through the equation (see, e.g., Ref. 24)

$$
\hbar^{2} Q^{2} / 2 m=2 E_{0}+\hbar \omega-2 \sqrt{E_{0}\left(E_{0}+\hbar \omega\right)} \cos \Phi .
$$

An incident wavelength of $6.3 \AA$ was used on IN16 and BSS. The measurements at IN16 covered an energy window from -15 to $15 \mu \mathrm{eV}$. The offset configuration of BSS was selected to cover a window from -29 to $1 \mu \mathrm{eV}$. The angular range extended from 11 to $149^{\circ}$ in IN16 and from 9 to $140^{\circ}$ in BSS. The HWHM of the instrumental resolution was $\approx 0.4$ $\mu \mathrm{eV}$ for IN16 and $\approx 1 \mu \mathrm{eV}$ for BSS. As the energy of the incident beam $(2.1 \mathrm{meV})$ is much larger than the maximum energy transfer, Eq. (13) can be well approximated by

$$
Q=\left(4 \pi / \lambda_{0}\right) \sin (\Phi / 2) \text {. }
$$

Thus, each angular value corresponds to a unique $Q$. The corresponding $Q$-range extended from 0.2 to $1.9 \AA^{-1}$.

A wavelength of $6.5 \AA$ was selected at IN5, yielding a resolution of HWHM of about $25 \mu \mathrm{eV}$. The angular range extended from 16 to $126^{\circ}$. The energy window ranged from -0.6 to $19.5 \mathrm{meV}$. At IN5 the approximation of Eq. (14) is 
(a)

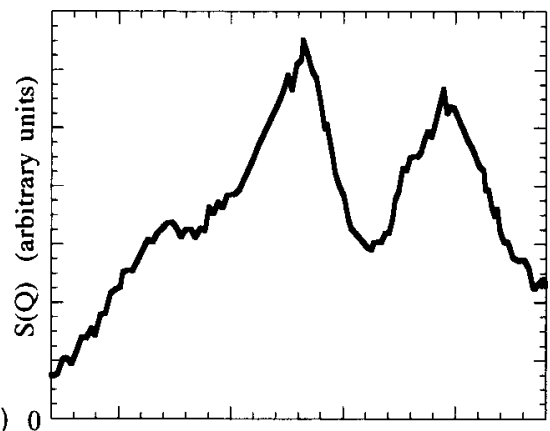

(b)

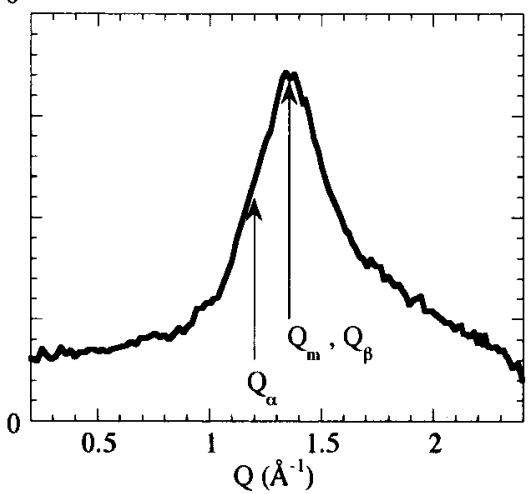

FIG. 1. $S(Q)$ of glassy ring-deuterated (a) and fully protonated (b) toluene. See the text for the meaning of $Q_{m}, Q_{\alpha}$, and $Q_{\beta}$.

not valid and the scattering function $S(Q, \omega)$ was obtained from $S(\Phi, \omega)$ by means of a standard interpolation program. We restricted the analysis to the quasielastic energy transfer from -1 to $2 \mathrm{meV}$. The $Q$-range obtained after this procedure extended from 0.7 to $1.7 \AA^{-1}$.

The coherent static intensities of the ring-deuterated sample and of a fully protonated one were determined on D7 by using spin polarization analysis. This allows for a separation of coherent and incoherent scattering by measuring both, neutron spin flip and nonspin flip intensities separately. ${ }^{25}$ $S(Q)$ for both samples is shown in Fig. 1.

\section{RESULTS}

Figures 2-4 show some of the experimental spectra at different sample temperatures and at the three spectrometers used in the investigation. As in the other structural glasses studied so far, the spectra at very low temperatures do not show defined tunneling peaks, but a broad feature, that results from a distribution of tunneling frequencies whose maximum lies below the instrumental resolution. ${ }^{11,12,16} \mathrm{We}$ followed the usual procedure to analyze the data in terms of the convolution of Eq. (12) with the instrumental resolutions. $^{26}$ A Gaussian form for the distribution $g\left(V_{3}\right)$ was first assumed

$$
g\left(V_{3}\right)=\left(1 / \sqrt{2 \pi} \sigma_{V}\right) \exp \left[-\left(V_{3}-V_{3_{0}}\right)^{2} / 2 \sigma_{V}^{2}\right],
$$

with $V_{3_{0}}$ the average barrier and $\sigma_{V}$ the standard deviation. Due to the independence of the pre-exponential factor $\Gamma_{\infty}$ on the barrier height, Eq. (6) establishes a linear dependence of $\ln \Gamma$ on $E_{A}$. As $E_{A}$ depends linearly on $V_{3}$ in good approximation, ${ }^{12}$ the distribution of classical activation energies $f\left(E_{A}\right)$ and classical Lorentzian broadenings $H(\ln \Gamma)$
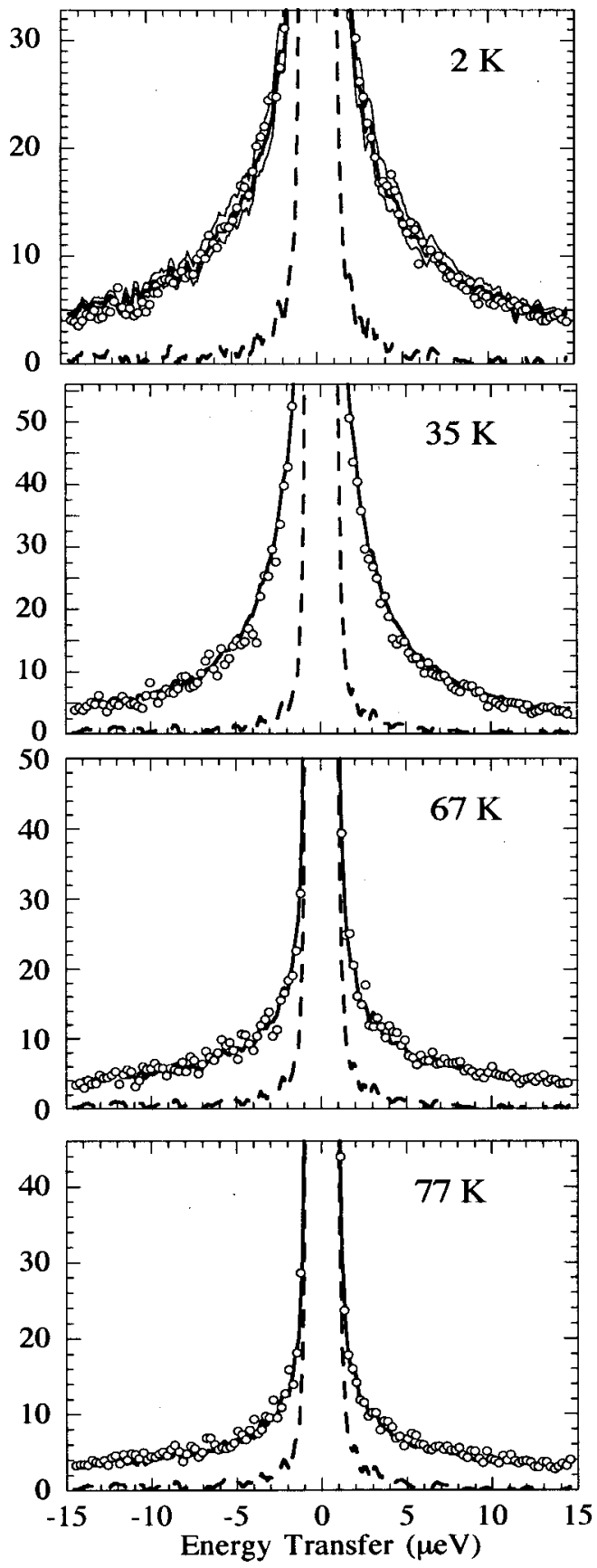

FIG. 2. IN16 experimental spectrum $S_{e}(Q, \omega)$ for methyl group dynamics in glassy toluene (points). The thick solid lines are the theoretical curves given by the RRDM, with the parameters $V_{3_{0}}=420 \mathrm{~K}, \sigma_{V}=200 \mathrm{~K}$, and $\Gamma_{\infty}$ $=8 \mathrm{meV}$. The thin solid lines in the spectrum at $2 \mathrm{~K}$ correspond to a description with $V_{3_{0}}=420 \mathrm{~K}$ and the two limiting values of the error bar of $\sigma_{V}, 180$, and $220 \mathrm{~K}$. The dashed line is the instrumental resolution, that has been rescaled to the maximum of $S_{e}(Q, \omega)$ at each temperature. $Q$ $=1.8 \AA^{-1}$. The scale is a $2.5 \%$ of the maximum for the spectrum at $2 \mathrm{~K}$ and a $5 \%$ for the others.

take the same functional form as $g\left(V_{3}\right)$, and are Gaussian in the present case. With all these considerations in mind, all the data obtained in the three instruments were fitted to the classical limit of the model, taking the classical form of Eq. (8): $S_{\mathrm{RRDM}}^{\mathrm{inc}}(Q, \omega)=\int H(\ln \Gamma) S_{\mathrm{MG}}^{\mathrm{inc}}\left(Q, \omega, V_{3}\right) d \ln \Gamma$, with $S_{\mathrm{MG}}^{\text {inc }}\left(Q, \omega, V_{3}\right)$ given by Eq. (7). This procedure enables calculation of the preexponential factor $\Gamma_{\infty}$, and the average 

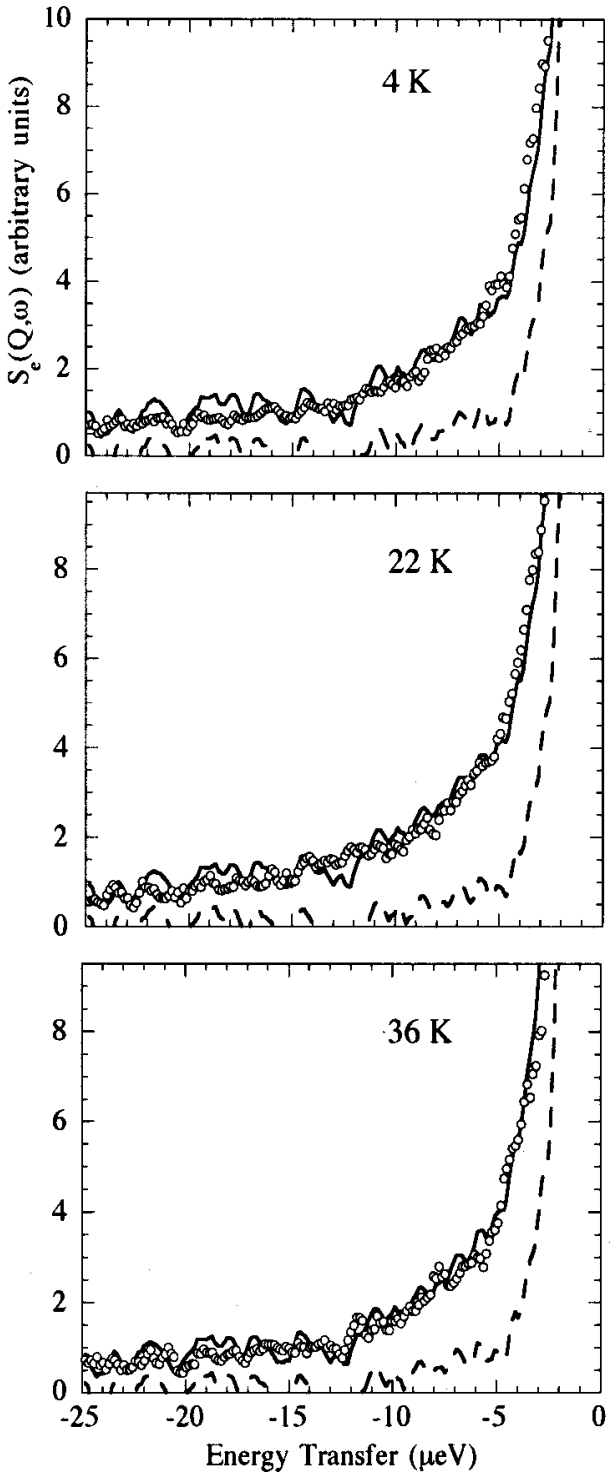

FIG. 3. As Fig. 2 for BSS. $Q=1.9 \AA^{-1}$. Scale: $10 \%$ of the maximum.

activation energy $E_{A_{0}}$ and the standard deviation $\sigma_{E}$ of $f\left(E_{A}\right)$ from fitting the series of average values $\ln \Gamma_{0}$ and standard deviations $\sigma$ of $H(\ln \Gamma)$ obtained at each temperature to the equations ${ }^{10,13}$

$$
\begin{aligned}
& \ln \Gamma_{0}=\ln \Gamma_{\infty}-\left[E_{A} / k T\right], \\
& \sigma=\sigma_{E} / k T .
\end{aligned}
$$

A discrete form of $H(\ln \Gamma)$ was taken in the calculations, with equally spaced values of $\ln \Gamma$ extending from $\ln \Gamma_{0}$ $-3 \sigma$ to $\ln \Gamma_{0}+3 \sigma$ (this corresponds to a distribution $f\left(E_{A}\right)$ extending from $E_{A_{0}}-3 \sigma_{E}$ to $E_{A_{0}}+3 \sigma_{E}$ ). The discrete function $H(\ln \Gamma)$ was normalized to unit area before constructing the theoretical scattering function.

As shown in Figs. 5 and 6, the values of $\ln \Gamma_{0}$ and $\sigma$ below $\approx 50 \mathrm{~K}$ deviate from the linear behavior given by Eqs. (16) and (17), indicating the breakdown of the classical model and the contribution of quantum effects below this temperature. Therefore, only the set of values $\left(\ln \Gamma_{0}, \sigma\right)$ obtained above $50 \mathrm{~K}$ was fitted to Eqs. (16) and (17). The
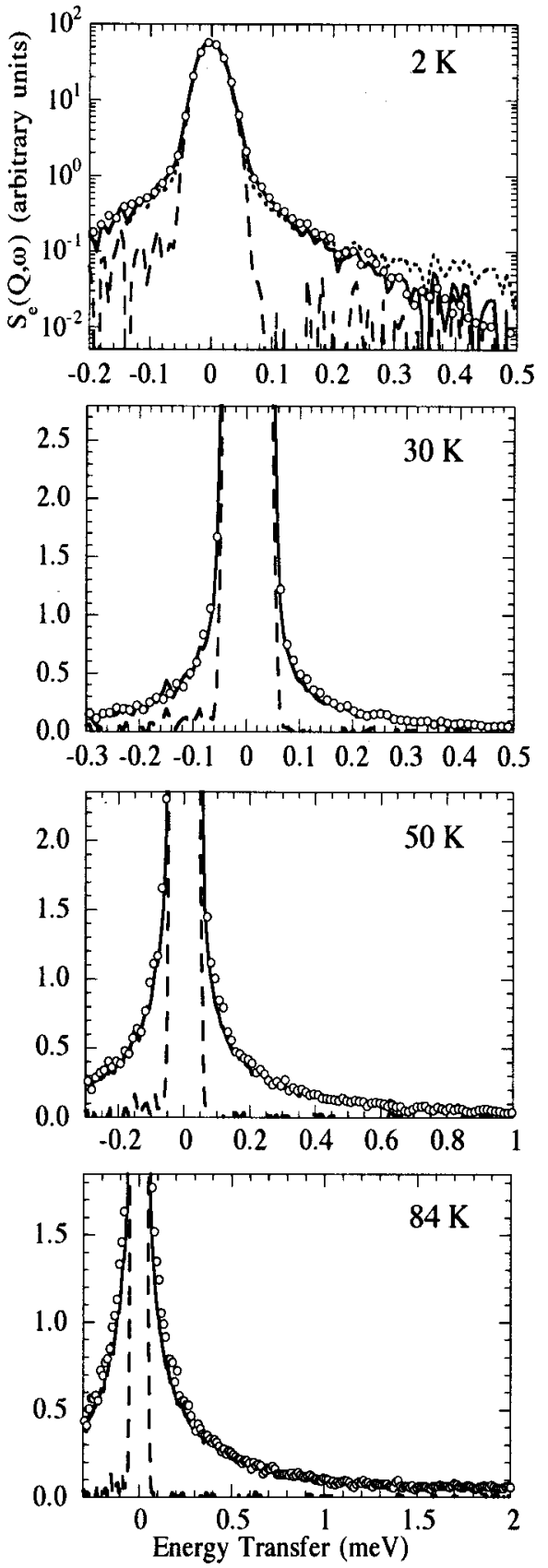

FIG. 4. As Fig. 2 for IN5. The spectrum at $2 \mathrm{~K}$ has been plotted in logarithmic scale. The dotted line is the theoretical curve given by an analysis in terms of a Gaussian distribution $g\left(V_{3}\right)$ (see the text). The scale for the other plots is $5 \%$ of the maximum. $Q=1.6 \AA^{-1}$.

values of the RRDM parameters obtained from such a fit were $\Gamma_{\infty}=7.2 \mathrm{meV}, E_{A_{0}}=340 \mathrm{~K}$, and $\sigma_{E}=205 \mathrm{~K}$. The two latter parameters yield an unphysical result: $f\left(E_{A}\right)$ presents a strong population of negative values of $E_{A}$ (from $E_{A_{0}}$ $-1.65 \sigma_{E}$ to $\left.E_{A_{0}}-3 \sigma_{E}\right)$. For this reason, a new analysis was made but imposing a cutoff at zero energy, and normalizing the new distributions to unit area. The new fit yielded, within the error bar, the same parameters as found above. It is worthy of remark that in this case, the parameters $E_{A_{0}}$ and $\sigma_{E}$ that characterize $f\left(E_{A}\right)$ do not correspond to the average activation energy and the standard deviation, since the distribution has been cutoff at zero energy. The actual values are 


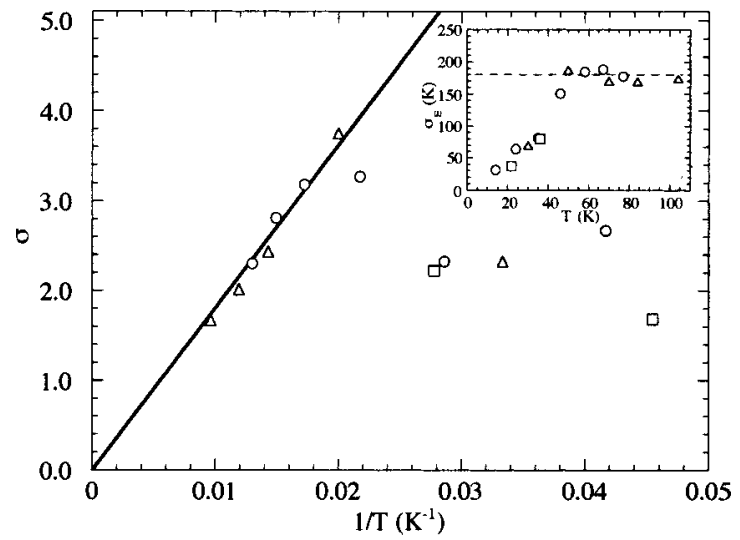

FIG. 5. Temperature dependence of the standard deviation $\sigma$ of the distribution $H(\ln \Gamma)$. Circles: IN16, squares: BSS, triangles: IN5. The line is a fit to Eq. (17). The inset shows the corresponding values of $\sigma_{E}$. The dashed line marks the average value.

$350 \mathrm{~K}$ for the average energy and $185 \mathrm{~K}$ for the standard deviation.

The eigenvalues of the Hamiltonian (1) were calculated by a standard diagonalization procedure for different values of $V_{3}$. The set of values $\left(V_{3}, E_{A}, E_{01}, \hbar \omega_{t}\right)$ obtained in this way allowed the transformation of the distribution $f\left(E_{A}\right)$ obtained in the high-temperature analysis into the distribution $g\left(V_{3}\right)$. The obtained parameters of $g\left(V_{3}\right)$ are $440 \mathrm{~K}$ for the average barrier and $195 \mathrm{~K}$ for the standard deviation (we remember that this latter values must not be confused with $V_{3_{0}}$ and $\sigma_{V}$, since as $f\left(E_{A}\right), g\left(V_{3}\right)$ is also a truncated Gaussian).

Once the RRDM parameters were determined, the theoretical scattering function (12) was calculated by taking the general expression (8) of $S_{\mathrm{RRDM}}^{\mathrm{inc}}(Q, \omega)$ instead of its classical limit. It must be noted that this latter calculation does not involve any fitting procedure, i.e., the spectra are simulated with the distribution $g\left(V_{3}\right)$ and the preexponential factor $\Gamma_{\infty}$ calculated in the high-temperature fitting procedure, and

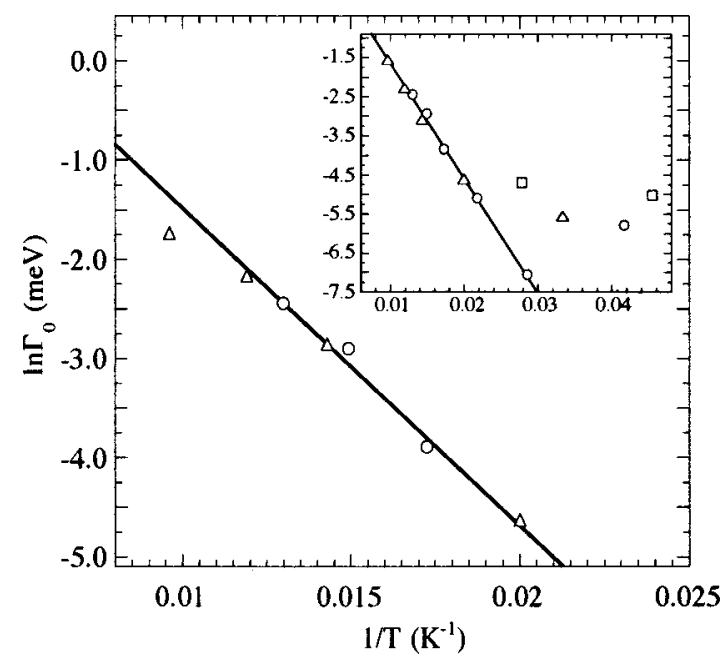

FIG. 6. Temperature dependence of the average value $\ln \Gamma_{0}$ of the distribution $H(\ln \Gamma)$, for a fixed value $\sigma_{E}=185 \mathrm{~K}$. Circles: IN16, squares: BSS, triangles: IN5. The inset show the values obtained in a fully free fit to the classical limit of the RRDM (see the text). Lines are fits to Eq. (16). compared with the experimental data. A good description of the spectra was achieved except for that of IN5 at $2 \mathrm{~K}$ (see the dotted line in the first plot of Fig. 4). Thus, the important population of very low barriers in $g\left(V_{3}\right)$ predicted a strong signal near the free rotor line at $0.655 \mathrm{meV}$, such signal not being observed in the IN5 spectra.

In order to avoid this problem, a new complete analysis was made in terms of a Gamma distribution

$$
\begin{aligned}
g\left(V_{3}\right)= & {\left[(p / e)^{p} / V_{3_{0}} \Gamma(p)\right] \times\left(V_{3} / V_{3_{0}}\right)^{p-1} } \\
& \times \exp \left[-p\left(V_{3}-V_{3_{0}}\right) / V_{3_{0}}\right],
\end{aligned}
$$

with $\Gamma(p)$ the Euler Gamma function and $p$ a dimensionless shape parameter. The standard deviation is obtained as $\sigma_{V}$ $=V_{3_{0}} / \sqrt{p}$. A Gamma distribution goes to zero at zero barrier height, avoiding the possibility of a strong population of quasi-free rotors (except for values of $p$ close to 1 , which result in nearly exponential distributions). We started the analysis as in the previous case, by fitting the hightemperature data to the classical limit of the RRDM. As $f\left(E_{A}\right)$ takes the same functional form as $g\left(V_{3}\right)$, it is straightforward to see that $f\left(E_{A}\right)$ is transformed through Eq. (16) into the distribution $H(\ln \Gamma)$ as

$$
\begin{aligned}
H(\ln \Gamma)= & {\left[(p / e)^{p} / \Gamma(p)\right] \frac{\left(\ln \Gamma_{\infty}-\ln \Gamma\right)^{p-1}}{\left(\ln \Gamma_{\infty}-\ln \Gamma_{0}\right)^{p}} } \\
& \times \exp \left[-p\left(\ln \Gamma_{0}-\ln \Gamma\right) /\left(\ln \Gamma_{\infty}-\ln \Gamma_{0}\right)\right] .
\end{aligned}
$$

The corresponding standard deviation is $\sigma=\left(\ln \Gamma_{\infty}\right.$ $\left.-\ln \Gamma_{0}\right) / \sqrt{p}$, that is again related to $\sigma_{E}$ through Eq. (17). The fits were done for different fixed values of $p$ to avoid the coupling of the fitting parameters. Figures 5 and 6 show the temperature dependence of $\sigma$ and $\ln \Gamma_{0}$ for a fixed value $p$ $=4.3$. Deviations from the linear behavior given by Eqs. (16) and (17) were observed below $\approx 50 \mathrm{~K}$, indicating the evidence of quantum effects.

The line in Fig. 5 corresponds to the fit of the data above this temperature to Eq. (17). The experimental data can be extrapolated to the origin. This result is consistent with the approximation of the independence of $\Gamma_{\infty}$ on $V_{3}$. If this approximation was not valid, we would have: $\ln \Gamma_{0}+x \sigma$ $=\ln \Gamma_{\infty}^{(x)}-\left[\left(E_{A_{0}}-x \sigma_{E}\right) / k T\right]$, with $\Gamma_{\infty}^{(x)}=\Gamma_{\infty}\left(V_{3_{0}}+x \sigma_{V}\right)$. Thus, from Eq. (16)

$$
\sigma=\left[\left(\ln \Gamma_{\infty}^{(x)}-\ln \Gamma_{\infty}\right) / x\right]+\left[\sigma_{E} / k T\right] .
$$

Taking into account that $x$ cannot be arbitrarily large (the whole area of the distribution is covered for values $|x| \sim 3$, $|x| \sim 4$ ), Eq. (17) is recovered only if $\ln \Gamma_{\infty}^{(x)} \approx \ln \Gamma_{\infty}$. The good agreement of the experimental results with Eq. (17) observed in all the systems studied up to now ${ }^{10-16}$ confirms the consistency of the latter approximation.

From the fit to Eq. (17), a value $\sigma_{E}=185 \mathrm{~K}$ was obtained. The inset in Fig. 5 shows the values of $\sigma_{E}$ calculated through Eq. (17) from the values of $\sigma$ at each temperature. The dashed line indicates the average value $\sigma_{E}=185 \mathrm{~K}$.

The fits of the experimental spectra were redone by fixing the values of $\sigma$ to those corresponding to $\sigma_{E}=185 \mathrm{~K}$, 


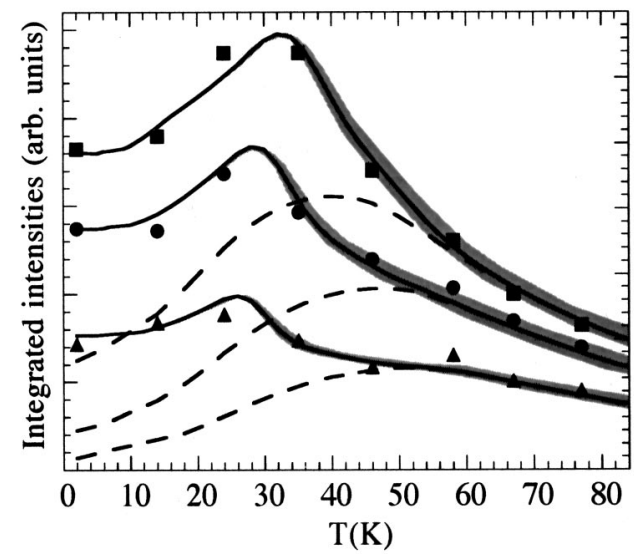

FIG. 7. Integrated IN16 intensities at $Q=1.8 \AA^{-1}$ in the ranges $1-3 \mu \mathrm{eV}$ (squares), 3-6.5 $\mu \mathrm{eV}$ (circles), and 6.5-10 $\mu \mathrm{eV}$ (triangles). Solid lines are the values predicted by the RRDM for the parameters $V_{3_{0}}=420 \mathrm{~K}, \sigma_{V}$ $=200 \mathrm{~K}$, and $\Gamma_{\infty}=8 \mathrm{meV}$. The shaded area corresponds to the different descriptions within the error bar of $\Gamma_{\infty}$, for the same parameters of $g\left(V_{3}\right)$. Dashed lines correspond to a description exclusively in terms of classical hopping.

and leaving $\ln \Gamma_{0}$ as the only fitting parameter. This procedure changed only slightly the values of $\ln \Gamma_{0}$ in the fully free fit (shown in the inset of Fig. 6). The line corresponds to the fit to Eq. (16). This fit yielded the values $E_{A_{0}}=320 \mathrm{~K}$ and $\Gamma_{\infty}=8 \mathrm{meV}$. The point at $105 \mathrm{~K}$ deviates from the linear behavior and was excluded from the fit. Such a deviation suggests the presence of other processes different from methyl group dynamics at this temperature, as it should be expected at temperatures close to the glass transition.

As in the previous case of the Gaussian distribution, $g\left(V_{3}\right)$ was determined from $f\left(E_{A}\right)$, and the spectra were simulated in the whole temperature range. An excellent description was achieved for all the spectra, including that of IN5 at $2 \mathrm{~K}$ (see solid thick lines in Figs. 2-4).

The above procedure was repeated for different values of the shape parameter $p$. Good descriptions were achieved only in the range $4<p<5$. The range of values for the RRDM parameters compatible with the spectra was: $V_{3_{0}}=420$ $\pm 20 \mathrm{~K}, \sigma_{V}=200 \pm 20 \mathrm{~K}$, and $\Gamma_{\infty}=8 \pm 2 \mathrm{meV}$. The uncertainties in the parameters of the distribution are illustrated in Fig. 2 in the spectrum at $2 \mathrm{~K}$. The solid thin lines correspond to the theoretical curves for $V_{3_{0}}=420 \mathrm{~K}$ and for the two limits of the error bar of $\sigma_{V}, 180$, and $220 \mathrm{~K}$.

The RRDM parameters obtained from the analysis in terms of a Gamma distribution are, within the error bars, equal to those obtained in the previous analysis in terms of a Gaussian distribution $g\left(V_{3}\right)$, showing that the RRDM parameters do not depend strongly on the functional form assumed for $g\left(V_{3}\right)$. It is worthy of remark that the value obtained for $\sigma_{V}$ is similar to those obtained in the different structural glasses analyzed up to now. ${ }^{10-16}$

We can show the suitability of the RRDM in the whole temperature range in a condensed way by plotting integrated intensities versus temperature. Figure 7 shows IN16 data for three different inelastic windows (points) together with the theoretical curves (solid lines) predicted by the RRDM parameters given above, $V_{3_{0}}=420 \mathrm{~K}, \sigma_{V}=200 \mathrm{~K}$, and $\Gamma_{\infty}$
$=8 \mathrm{meV}$. The shaded area corresponds to the descriptions with the same parameters for $g\left(V_{3}\right)$ and $\Gamma_{\infty}$ ranging from 6 to $10 \mathrm{meV}$, i.e., within its uncertainty band given above. The curves were damped by the Debye-Waller factor of cubic lattice, $\exp \left(-2 Q^{2}\left\langle u^{2}\right\rangle / 3\right)$, to give account for the intensity decay due to vibrations. A mean-squared displacement $\left\langle u^{2}\right\rangle$ $=3 \times 10^{-4}$ T was assumed. ${ }^{8}$

For comparison, a description exclusively in terms of classical hopping (dashed lines) has been extrapolated to low temperature. The excess of intensity below $T \approx 50 \mathrm{~K}$ not reproduced by the classical limit of the RRDM, results from methyl groups having not reached the classical hopping regime at these temperatures.

\section{DISCUSSION}

When comparing the methyl group dynamics in the glassy and in the crystalline state of toluene, a first problem arises, since crystalline toluene can exist in two phases: $:^{20,27}$ A stable phase ( $\alpha$-phase) and a metastable one ( $\beta$-phase). The latter can be obtained by heating up the supercooled liquid to $T \approx 130 \mathrm{~K}$. From early $\mathrm{X}$-ray measurements ${ }^{27}$ it is known that the short-range structure of the glass shows features rather similar to those of the $\beta$-phase. This is confirmed by the measured coherent static intensity of the fully protonated glassy sample [Fig. 1(b)]. Due to the high mass and coherent cross section of the carbon in comparison with the proton (5.6 barns for carbon while only 1.8 barns for proton), this function corresponds approximately to the Fourier transform of the center-of-mass pair-correlation function $F(r)$. The maximum at $Q_{m}=1.35 \AA^{-1}$ coincides with the value $Q_{\beta}=2 \pi / R_{\beta}$, with $R_{\beta}=4.7 \AA$, the first maximum of $F(r)$ for $\beta$-toluene deduced from X-ray measurements. ${ }^{27}$ The corresponding maximum for the $\alpha$-phase is $R_{\alpha}=5.3 \AA{ }^{27}$ yielding a value $Q_{\alpha}=1.20 \AA^{-1}$, far from $Q_{m}$. Therefore, the comparison has to be made with the $\beta$-phase as the crystalline reference phase.

In the past, investigations on methyl group dynamics in toluene have concentrated on the stable crystalline $\alpha$-phase. There are two nonequivalent methyl groups ${ }^{27}$ in the unit cell of the $\alpha$-phase, with two different barriers. These barriers are well determined by early neutron scattering ${ }^{28}$ and $\mathrm{NMR}^{29}$ measurements. An expansion including a second Fourier term, ${ }^{28} V(\Phi)=V_{3}(1-\cos 3 \Phi) / 2+V_{6}[1-\cos (6 \Phi+\delta)] / 2$, was needed to give an accurate description of the experimental data. One of the barriers (we will refer to the corresponding methyl group as $\alpha$-I), is basically threefold $\left(V_{3}\right.$ $\left.=267 \mathrm{~K}, V_{6}=-21 \mathrm{~K}, \delta=35^{\circ}\right)$ while the other one $(\alpha$-II $)$, presents a strong sixfold component $\left(V_{3}=346 \mathrm{~K}, \quad V_{6}\right.$ $=-173 \mathrm{~K}, \delta=17^{\circ}$ ). The corresponding classical activation energies, which can be better related to the degree of hindrance, are $E_{A}(\alpha-\mathrm{I})=210 \mathrm{~K}$ and $E_{A}(\alpha-\mathrm{II})=340 \mathrm{~K}$. On the contrary, in the $\beta$-phase all the methyl groups are equivalent. ${ }^{27}$ To our knowledge, the only existing information about the rotational barrier in the $\beta$-phase is an old measurement of the tunneling frequency, $\hbar \omega_{t}=88 \mu \mathrm{eV} .{ }^{30}$ For a purely threefold potential, this value corresponds to a barrier $V_{3}=167 \mathrm{~K}$. The corresponding classical activation energy is $E_{A}(\beta)=120 \mathrm{~K}$. 


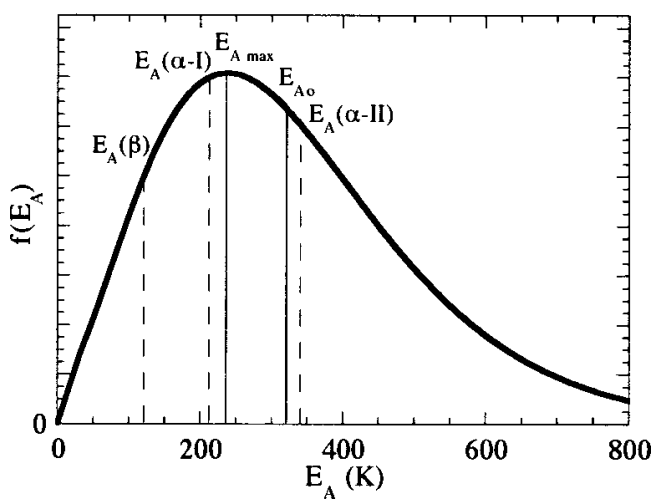

FIG. 8. Distribution of classical activation energies $f\left(E_{A}\right)$ deduced from the RRDM parameters given in the text. The maximum and average energy of the distribution have been indicated together with the values in the crystalline phases.

Figure 8 shows the comparison between the classical activation energies in the crystalline phases and the distribution $f\left(E_{A}\right)$ that has been calculated above for the glass. The average energy of this distribution, $E_{A_{0}} \approx 320 \mathrm{~K}$, is about $15 \%$ higher than the average value of the $\alpha$-phase, $275 \mathrm{~K}$, and much higher (about 165\%) than the unique value of 120 $\mathrm{K}$ of the reference $\beta$-phase. At this point we remember that this latter value was deduced in order to reproduce the experimental value of the tunneling frequency $\hbar \omega_{t}=88 \mu \mathrm{eV}$, on the assumption of a purely threefold rotational potential. A sixfold contribution to the potential in the $\beta$-phase could be obtained by measuring the librational peaks or by following the evolution of the tunneling spectrum with increasing temperature. Unfortunately, these latter measurements could not be done due to the limited time of the experiment and the difficulty to keep the metastable $\beta$-phase for a time sufficiently long, this one decaying irreversibly in the stable $\alpha$-phase. Though we cannot discard sixfold contributions, it can be seen by mapping the space $\left(V_{3}, V_{6}, \delta\right)$ and calculating the level scheme for the corresponding $V(\Phi)$, that the value of $\hbar \omega_{t}=88 \mu \mathrm{eV}$ is incompatible with values of $E_{A}$ higher than $\approx 230 \mathrm{~K}$, still about $40 \%$ below the average value $E_{A_{0}}$ in the glass. Therefore, though the shape of the rotational potential in the $\beta$-phase is still not well determined, we can conclude that the barrier is notably weaker than in the glass. It is worthy of remark that a similar effect was observed in ethylbenzene, ${ }^{31}$ a system similar to toluene. In this system, a broadening and a shift to higher energy was observed for the first librational peak of the glassy sample when compared to that of the crystal, suggesting a distribution of rotational potentials with an average barrier higher than the unique barrier in the crystal.

\section{CONCLUSIONS}

We have carried out a detailed neutron scattering study of methyl group dynamics in glassy toluene, a system where the rotational barrier is of mainly intermolecular origin. The transition from quantum rotational tunneling to classical hopping has been investigated by using three different spectrom- eters, covering an energy range from $0.5 \mu \mathrm{eV}$ to $2 \mathrm{meV}$. The spectra were analyzed in terms of the RRDM. An asymmetric Gamma function $g\left(V_{3}\right)$ achieved a description of the experimental spectra better than the usual Gaussian form, that failed for very low barriers in the tunneling regime. The RRDM parameters were accurately determined, taking the values of $8 \mathrm{meV}$ for the preexponential factor $\Gamma_{\infty}, 200 \mathrm{~K}$ for the standard deviation $\sigma_{V}$ and $420 \mathrm{~K}$ for the average barrier $V_{3_{0}}$ of $g\left(V_{3}\right)$. This latter value is much higher than the unique barrier in the crystalline $\beta$-phase, which short-range structure is similar to that of the glassy state. Although a simple explanation for this result cannot be envisaged, this effect likely indicates that the intermolecular contribution to the rotational barrier of the methyl group in toluene is strongly affected by structural disorder. Molecular dynamics simulations could shed new light on this question.

\section{ACKNOWLEDGMENTS}

This work has been supported by the projects DGICYT, PB97-0638; GV, EX 1998-23; UPV/EHU, 206.215-G20/98. The support from DIPC and Iberdrola S. A. are also acknowledged. One of the authors (A.J.M.) acknowledges a grant from the Basque Government. The authors thank A. Arbe, J. R. Stewart, and A. Murani for experimental help and F. Álvarez for some calculations.

${ }^{1}$ W. Press, Single-Particle Rotations in Molecular Crystals, Springer Tracts in Modern Physics (Springer, Berlin, 1981), Vol. 92.

${ }^{2}$ C. J. Carlile and M. Prager, Int. J. Mod. Phys. B 7, 3113 (1993).

${ }^{3}$ M. Prager and A. Heidemann, Chem. Rev. 97, 2933 (1997).

${ }^{4}$ A. Heidemann, M. Prager, and M. Monkenbusch, Z. Phys. B: Condens. Matter 76, 77 (1989).

${ }^{5}$ M. R. Johnson, M. A. Neumann, B. Nicolai, P. Smith, and G. J. Kearley, Chem. Phys. 215, 343 (1997).

${ }^{6}$ M. R. Johnson, M. Prager, H. Grimm, M. A. Neumann, G. J. Kearley, and C. C. Wilson, Chem. Phys. 244, 49 (1999).

${ }^{7}$ F. Álvarez, A. Alegría, J. Colmenero, T. M. Nicholson, and G. R. Davies, Macromolecules 33, 8077 (2000)

${ }^{8}$ B. Frick and L. J. Fetters, Macromolecules 27, 974 (1994).

${ }^{9}$ R. M. Dimeo and D. A. Neumann, Phys. Rev. B 63, 014301 (2001).

${ }^{10}$ A. Chahid, A. Alegría, and J. Colmenero, Macromolecules 27, 3282 (1994).

${ }^{11}$ J. Colmenero, R. Mukhopadhyay, A. Alegría, and B. Frick, Phys. Rev. Lett. 80, 2350 (1998).

${ }^{12}$ A. J. Moreno, A. Alegría, J. Colmenero, and B. Frick, Phys. Rev. B 59, 5983 (1999)

${ }^{13}$ R. Mukhopadhyay, A. Alegría, J. Colmenero, and B. Frick, Macromolecules 31, 3985 (1998).

${ }^{14}$ A. J. Moreno, A. Alegría, and J. Colmenero, Phys. Rev. B 63, 060201 (2001).

${ }^{15}$ A. J. Moreno, A. Alegría, J. Colmenero, and B. Frick, Macromolecules 34, $4886(2001)$

${ }^{16}$ A. J. Moreno, A. Alegría, J. Colmenero, and B. Frick, ILL Exp. Rep. 6-05-467 (2001)

${ }^{17}$ S. Clough, A. Heidemann, and M. N. J. Paley, J. Phys. C 14, 1001 (1981).

${ }^{18} \mathrm{~F}$. Álvarez (private communication).

${ }^{19}$ M. Prager, M. Monkenbusch, R. M. Ibberson, W. I. F. David, and D. Cavagnat, J. Chem. Phys. 98, 5653 (1993).

${ }^{20}$ J. Bruneaux-Poulle, L. Bossio, and M. Dupont, J. Chim. Phys. Phys.Chim. Biol. 76, 333 (1979).

${ }^{21}$ A. C. Hewson, J. Phys. C 15, 3841 (1982).

${ }^{22}$ A. Würger, Z. Phys. B: Condens. Matter 76, 65 (1989).

${ }^{23}$ M. Prager, K. H. Duprée, and W. Müller-Warmuth, Z. Phys. B: Condens. Matter 51, 309 (1983). 
${ }^{24}$ T. Springer, Quasielastic Neutron Scattering for the Investigation of Diffusive Motions in Solids and Liquids, Springer Tracts in Modern Physics (Springer, Berlin, 1972), Vol. 64.

${ }^{25}$ O. Schärpf and H. Capellmann, Phys. Status Solidi A 135, 359 (1993).

${ }^{26} \mathrm{~A}$ flat background was introduced as an extra parameter in the analysis of the IN5 data to give account for the low-frequency vibrations expected in this energy window.
${ }^{27}$ J. Bruneaux-Poulle, A. Defrain, and M. Dupont, J. Chim. Phys. Chim. Phys. Biol. 78, 217 (1981).

${ }^{28}$ D. Cavagnat, A. Magerl, C. Vettier, and S. Clough, J. Phys. C 19, 6665 (1986).

${ }^{29}$ D. van der Putten, G. Diezemann, F. Fujara, K. Hartmann, and H. Sillescu, J. Chem. Phys. 96, 1748 (1992).

${ }^{30}$ D. Cavagnat and W. Petry, ILL Exp. Rep. 09-03-566 (1989).

${ }^{31}$ B. Frick, J. Williams, S. Trevino, and R. Erwin, Physica B 213-214, 506 (1995). 